\title{
Contribution of Starlette, Stella, and AJISAI to the SLR-derived global reference frame
}

\author{
Krzysztof Sośnica · Adrian Jäggi · Daniela Thaller • \\ Gerhard Beutler • Rolf Dach
}

Received: 9 September 2013 / Accepted: 10 April 2014 / Published online: 13 May 2014

(C) Springer-Verlag Berlin Heidelberg 2014

\begin{abstract}
The contribution of Starlette, Stella, and AJISAI is currently neglected when defining the International Terrestrial Reference Frame, despite a long time series of precise SLR observations and a huge amount of available data. The inferior accuracy of the orbits of low orbiting geodetic satellites is the main reason for this neglect. The Analysis Centers of the International Laser Ranging Service (ILRS ACs) do, however, consider including low orbiting geodetic satellites for deriving the standard ILRS products based on LAGEOS and Etalon satellites, instead of the sparsely observed, and thus, virtually negligible Etalons. We process ten years of SLR observations to Starlette, Stella, AJISAI, and LAGEOS and we assess the impact of these Low Earth Orbiting (LEO) SLR satellites on the SLR-derived parameters. We study different orbit parameterizations, in particular different arc lengths and the impact of pseudostochastic pulses and dynamical orbit parameters on the quality of the solutions. We found that the repeatability of the East and North components of station coordinates, the quality of polar coordinates, and the scale estimates of the reference are improved when combining LAGEOS with low orbiting SLR satellites. In the multi-SLR solutions, the scale and the $Z$ component of geocenter coordinates are less affected by deficiencies in solar radiation pressure modeling than in the LAGEOS-1/2 solutions, due to substantially reduced correlations between the $Z$ geocenter coordinate and empirical orbit parameters. Eventually, we found that the stan-
\end{abstract}

K. Sośnica (凶) · A. Jäggi · D. Thaller · G. Beutler · R. Dach Astronomical Institute, University of Bern, Sidlerstrasse 5, 3012 Bern, Switzerland e-mail: krzysztof.sosnica@aiub.unibe.ch; sosnica@aiub.unibe.ch

Present Address:

D. Thaller

Bundesamt für Kartographie und Geodäsie, Richard-Strauss-Allee 11, 60598 Frankfurt am Main, Germany dard values of Center-of-mass corrections (CoM) for geodetic LEO satellites are not valid for the currently operating SLR systems. The variations of station-dependent differential range biases reach 52 and $25 \mathrm{~mm}$ for AJISAI and Starlette/Stella, respectively, which is why estimating stationdependent range biases or using station-dependent CoM, instead of one value for all SLR stations, is strongly recommended. This clearly indicates that the ILRS effort to produce CoM corrections for each satellite, which are site-specific and depend on the system characteristics at the time of tracking, is very important and needs to be implemented in the SLR data analysis.

Keywords Satellite geodesy $\cdot$ SLR $\cdot$ LAGEOS $\cdot$ Starlette AJISAI - Stella $\cdot$ Precise orbit determination $\cdot$ Reference frame $\cdot$ Geocenter

\section{Introduction}

\subsection{SLR geodetic satellites}

The spherical geodetic satellites have greatly contributed to high-precise space geodesy in the last decades. Starlette was the first satellite with a minimized area-to-mass ratio, due to a dense core made by Uranium, and thus, with minimized non-gravitational forces perturbing the satellite orbit. Starlette was launched in 1975 by the French Space Agency and defined together with the Laser Geodynamics Satellite (LAGEOS-1), launched one year later, the advent of a new era in Satellite Laser Ranging (SLR). For many years, the SLR observations to Starlette and LAGEOS- 1 allowed determining positions and motions of SLR stations (and thus also the plate motions), Earth Rotation Parameters (ERP), the global scale, and the tidal displacements with a great precision (e.g., 
Table 1 Characteristics of geodetic satellites and their orbits

\begin{tabular}{|c|c|c|c|c|c|}
\hline & AJISAI & Starlette & Stella & LAGEOS-1 & LAGEOS-2 \\
\hline Diameter (m) & 2.15 & 0.24 & 0.24 & 0.60 & 0.60 \\
\hline Mass (kg) & 685 & 47 & 48 & 407 & 405 \\
\hline Area-to-mass $\left(\mathrm{m}^{2} / \mathrm{kg}\right)$ & $58.0 \mathrm{e}-4$ & $9.6 e-4$ & $9.4 \mathrm{e}-4$ & $6.9 \mathrm{e}-4$ & $7.0 \mathrm{e}-4$ \\
\hline Radiation coeff. $C_{R}$ & 1.03 & 1.134 & 1.131 & 1.13 & 1.13 \\
\hline A priori drag scaling factor $C_{D}$ & 2.80 & 2.37 & 2.37 & - & - \\
\hline Semi-major axis $(\mathrm{km})$ & 7,866 & 7,334 & 7,176 & 12,274 & 12,158 \\
\hline Orbit altitude (km) & 1,500 & $800-1,100$ & 830 & 5,860 & 5,620 \\
\hline Eccentricity & 0.0016 & 0.0205 & 0.0010 & 0.0039 & 0.0137 \\
\hline Inclination $\left(^{\circ}\right)$ & 50.04 & 49.84 & 98.57 & 109.90 & 52.67 \\
\hline Draconitic year (days) & 89 & 73 & 182 & 560 & 222 \\
\hline A priori sigma $(\mathrm{mm})$ & 25 & 20 & 20 & 10 & 10 \\
\hline A priori CoM correction (mm) & $1,010^{1}$ & $78^{2}$ & $78^{2}$ & Station-spec. CoM ${ }^{3}$ & Station-spec. CoM ${ }^{3}$ \\
\hline
\end{tabular}

${ }^{1}$ Standard CoM value (http://ilrs.gsfc.nasa.gov/missions/satellite_missions/current_missions/ajis_com.html)

${ }^{2}$ Ries (2008)

${ }^{3}$ Appleby et al. (2012)

Smith and Turcotte 1993; Cheng et al. 1990). The constellation of geodetic SLR satellites was extended by the launch of AJISAI (also called the Experimental Geodetic Payload), LAGEOS-2, and Stella in 1986, 1992, and 1993, respectively. LAGEOS-2 and Stella are twins of LAGEOS-1 and Starlette, respectively, with similar altitudes and area-to-mass ratios, but with different inclinations of the satellite orbits (see Table 1). The area-to-mass ratio of AJISAI is larger than the value of the other aforementioned satellites, because AJISAI was designed not only for SLR, but also for optical observations (Sengoku 1998). Therefore, the satellite is equipped with curved mirrors covering the large satellite surface in addition to the embedded laser-dedicated retroreflectors.

Schutz et al. (1989) demonstrated the potential of Starlette for the determination of ERP. At that time, the best pole coordinates from Starlette agreed within 4.4 and 3.6 mas for the $X$ and $Y$ coordinates of the pole, respectively, with values obtained from LAGEOS-1 observations. In a recent analysis, Gourine (2012) demonstrated that ERP obtained from a combined solution containing LAGEOS-1 and LAGEOS-2 are better than those from a combined LAGEOS-1 and Starlette solution. A combined LAGEOS-1/2 - Starlette solution was, however, not considered. Thus, an improvement of a combined multi-satellite solution has not yet been demonstrated.

Lejba and Schillak (2011) showed that Starlette, Stella, and AJISAI can be used for the determination of SLR station coordinates and velocities, however with an inferior quality compared to the LAGEOS solutions. The analysis revealed artifacts of the vertical component of station coordinates when using AJISAI data. For some SLR stations, the vertical component is systematically shifted w.r.t. the vertical component obtained from LAGEOS or Starlette/Stella solutions.
Otsubo et al. (1999) and Otsubo and Appleby (2003) studied the Center-of-Mass corrections (CoM) of AJISAI, LAGEOS, and Etalon and showed that applying one CoM correction for all SLR stations is not sufficient for large SLR satellites. They concluded that the CoM should be applied station-specific, because of differences in equipments and screening procedures at SLR stations.

In 2012, a new geodetic satellite-the Laser Relativity Satellite (LARES) was launched (Paolozzi and Ciufolini 2013). LARES has the smallest area-to-mass ratio of all satellites in orbit. We do not study the contribution of LARES, because of the relatively short time span of SLR observations as compared to other geodetic satellites. First results of LARES-derived reference frame can be found, e.g., in Sośnica et al. (2014).

\subsection{Goal of the analysis}

Up till now, the contributions of Starlette, Stella, and AJISAI have completely been neglected when defining the International Terrestrial Reference Frame (ITRF, Altamimi et al. 2011), despite the huge amount of data collected in a long time span. International Laser Ranging Service (ILRS, Pearlman et al. 2002) routinely generates products based on high orbiting LAGEOS and Etalon satellites, whereas geodetic Low Earth Orbiters (LEO) do not contribute to the ILRS solutions. The SLR-derived parameters and the SLR part of the ITRF are, therefore, almost solely defined by LAGEOS-1 and LAGEOS-2, because the contribution of the very high orbiting Etalon satellites is virtually negligible (Thaller et al. 2014b).

Currently, the ILRS ACs consider the possibility of including low orbiting geodetic satellites to the standard ILRS prod- 
ucts (e.g., LARES, Starlette) instead of including the sparsely observed Etalons ${ }^{1}$. One may ask whether the SLR-derived reference frame and ERP can be improved by incorporating SLR observations to Starlette, Stella, and AJISAI?

We study the potential of combining many SLR satellites with different orbital characteristics by adopting a parameterization of SLR LEO orbits based on pseudo-stochastic pulses (instantaneous velocity changes at particular epochs in three directions applied to satellite orbits with predefined sigmas). We use latest state-of-the-art models according to the IERS Conventions 2010 (Petit and Luzum 2011). 10 years of SLR data are homogeneously processed using the development version 5.3 of the Bernese GNSS Software (Dach et al. 2007). Special emphasis is put on the aspects related to orbit parameterization, the impact of LEO data on the estimation of geocenter coordinates, ERP, and the station coordinates.

The Bernese GNSS Software was originally designed for processing GNSS data, but was recently extended by the capability of processing SLR data to high orbiting spherical satellites (Thaller et al. 2011). For the purpose of this paper, the capability of processing low orbiting spherical satellites was added to the Bernese GNSS Software.

\section{Orbit modeling}

Precise orbit determination of geodetic LEOs such as Starlette, Stella, and AJISAI is more demanding than the determination of the LAGEOS orbits, because of:

- A larger sensitivity to the Earth gravity field and to its temporal variations,

- Deficiencies in air drag models and variations of atmosphere density in the upper atmosphere,

- Deficiencies of SLR station-specific range biases due to different laser systems used at SLR stations.

The issue of uncertainties and the sensitivity to the timevarying Earth gravity field is handled using the Earth gravity field model EGM2008 (Pavlis et al. 2012) based mostly on data of the GRACE mission, and the estimation of timevariable low-degree gravity field coefficients from the SLR data. EGM2008 is used up to degree 30 for LAGEOS (Sośnica et al. 2012a) and up to degree 90 for the LEOs. Some of the low-degree harmonics are not taken from the model, but are replaced by the values from the IERS Conventions 2010 (Petit and Luzum 2011). This includes the application of drifts for the low-degree zonal coefficients up to degree 4.

\footnotetext{
$\overline{1}$ http://ilrs.gsfc.nasa.gov/docs/2012/AWG_Minutes_Frascati_2012. pdf.
}

Deficiencies in atmospheric drag models are absorbed by estimating empirical and stochastic orbit parameters (see Sect. 2.3).

The issue of poorly known CoM corrections for LEOs is addressed by estimating station- and satellite-specific range biases and by combining LEO and LAGEOS solutions. The CoM corrections for LAGEOS satellites are well established and no range biases for most SLR stations have to be estimated in the LAGEOS solutions. Therefore, the station- and LEO-specific range biases may compensate for inadequate values of CoM corrections of LEO satellites. Moreover, the resulting combined solutions are entirely consistent with the LAGEOS solutions, because the scale is derived from LAGEOS tracking data to the greatest extent.

\subsection{Orbit parameterization}

The satellite orbits are defined by a set of six osculating orbital elements referring to the initial epoch of the arc, up to nine dynamical orbit parameters, and by pseudo-stochastic pulses (Beutler et al. 1994). The dynamical orbit parameters absorb unmodeled and mismodeled gravitational and non-gravitational forces perturbing the satellite orbits. They are represented by constant accelerations and/or once-perrevolution (OPR) terms in three directions, i.e., radial $(R)$, out-of-plane $(W)$ and along-track $(S)$. The angular argument associated with OPR accelerations is $u$, i.e., the argument of latitude. Typically, a subset of these parameters is used for the precise orbit determination (see Sects. 2.2. and 2.3). The pseudo-stochastic pulses are velocity changes imposed on the satellite orbits acting in the aforementioned pre-defined directions at pre-defined epochs. They are constrained to zero with user-defined a priori standard deviations. The pseudostochastic pulses compensate for unmodeled accelerations and they assure the continuity of the orbit. Pseudo-stochastic orbit parameters are used at the Center for Orbit Determination in Europe (CODE, Dach et al. 2009) not only for the determination of GNSS orbits, but also for the determination of orbits of LEO satellites (Jäggi et al. 2006) and for gravity field determination using, e.g., GRACE data (Beutler et al. 2010).

\subsection{LAGEOS orbits}

Table 1 shows the characteristics of geodetic satellites. Analysis specifications are provided in Table 2.

The dynamical $\mathrm{S}_{0}$ parameter (constant acceleration in along-track) absorbs in particular the unmodeled Yarkovsky and the Yarkovsky-Schach thermal effects (Rubincam 1998), which are responsible for the decay of the semi-major axis of the LAGEOS satellites. The OPR sine/cosine terms (SS/SC) absorb the in-plane orbit perturbations, caused by mismodeled direct and indirect solar radiation pressure, unmodeled 
Table 2 Parameters estimated in LAGEOS and LEO solutions

\begin{tabular}{lll}
\hline Parameter & LAGEOS-1/2 & LEO \\
\hline Station coordinates & Weekly & Weekly \\
Earth rotation parameters & PWL daily & PWL daily \\
Geocenter coordinates & Weekly & Weekly \\
Gravity field & Up to d/o 3 & Up to d/o 3 \\
Range biases & Selected sites & All sites \\
Satellite orbits & & \\
Osculating elements & Weekly & Weekly \\
Constant along-track $S_{0}$ & Weekly & - \\
Air drag scaling factor & - & Daily \\
Once-per-rev SS, SC & Weekly & Daily \\
Once-per-rev WS, WC & - & Daily \\
Pseudo-stochastic pulses & - & OPR in $S$ \\
\hline
\end{tabular}

thermal forces, and the asymmetric LAGEOS reflectivity (Métris et al. 1997). As opposed to the typical orbit parameterization of the ILRS $\mathrm{ACs}^{2}$, the OPR terms in out-ofplane are not estimated, because of the correlation between piecewise linear (PWL) Length-of-Day (LoD), $C_{20}$, and the W OPR term acting on the entire arc (Sośnica et al. 2012a; Jäggi et al. 2012).

\subsection{LEO orbits}

In the Starlette, Stella, and AJISAI (LEO) 7-day solutions, we apply the model NRLMSISE-00 (Picone et al. 2002) for the atmospheric drag and we estimate daily scaling factors instead of a constant acceleration in along-track $\left(\mathrm{S}_{0}\right.$, see Table 2).

The OPR dynamical parameters together with pseudostochastic pulses account for large orbit perturbations due to variations of the atmosphere density in the upper atmosphere. In analogy to the LAGEOS satellites, the dynamical parameters account also for the mismodelled forces due to direct solar radiation pressure, indirect radiation pressure (albedo), and thermal effects for Starlette, Stella, and AJISAI. Pseudostochastic pulses are estimated at intervals of one revolution period in the along-track direction with an a priori standard deviation of $10^{-7} \mathrm{~m} / \mathrm{s}$.

We estimate range biases for all SLR stations, because of the lack of precise CoM corrections for LEO. Therefore, the scale in LEO-only solutions suffers from a rank deficiency, whereas in the combined LAGEOS-LEO solution the scale is defined almost uniquely by LAGEOS. The LAGEOS-derived scale is of superior quality due to much less perturbed orbits and the well-established CoM corrections (Appleby et al. 2012). We adopted the standard a priori CoM correction (see

\footnotetext{
${ }^{2} \mathrm{ftp} / / /$ cddis.gsfc.nasa.gov/pub/slr/products/ac/bkg.dsc.
}

Table 1) for AJISAI, whereas the CoM corrections for Starlette and Stella are taken from Ries (2008).

\subsection{Solution set-up}

As recommended by the IERS Conventions 2010, the atmospheric tidal loading with $S_{1}, S_{2}$ constituents is applied. In addition, we apply the non-tidal atmospheric loading station corrections to remove the Blue-Sky effect and the associated scale deficiency of $0.2 \mathrm{ppb}$ (Sośnica et al. 2013).

SLRF $2008^{3}$ - the ILRS realization of ITRF2008 (Altamimi et al. 2011) serves as a priori reference frame. The datum is defined in every solution by imposing the no-net-rotation and no-net-translation minimum conditions. For the datum definition, the SLR core sites are selected as recommended by the ILRS. The ILRS-recommended data handling file 4 is used, as well. The data handling file is maintained by the ILRS and contains, e.g., range biases and requested data exclusions. The set of core stations is verified in every week by removing the core stations with RMS of residuals from the Helmert transformation larger than $25 \mathrm{~mm}$ for at least one component. Stations observing only one pass of one satellite are also rejected from the datum definition.

The direct and indirect solar radiation pressure (Earth's albedo and Earth's infrared emissivity) are taken into account by assuming the satellites to be homogeneous spheres. The radiation pressure coefficient $C_{R}$ is fixed to the values from Table 1. The specularity of Earth's surface is neglected for the albedo reflectivity (Rodriguez-Solano et al. 2012).

We use the PWL ERP parameterization (Thaller et al. 2014b), in which the polar motion and UT1-UTC are represented by polygons, and therefore, they are continuous at the day boundaries. UT1-UTC is fixed to the a priori IERS-08$\mathrm{C} 04$ series at the day boundary between the third and fourth day, to avoid correlations with the satellite ascending nodes. Thaller et al. (2014b) show that the PWL parameterization is more effective for ERP determination in LAGEOS solutions than the piecewise constant ERP parameterization, in particular in a simultaneous estimation of $\mathrm{LoD}$ and $C_{20}$.

The adopted a priori observation sigmas from the variance analysis of observation residuals are 10,20 , and $25 \mathrm{~mm}$ for LAGEOS-1/2, Starlette/ Stella, and AJISAI normal point observations, respectively (see Table 1).

\section{Solution statistics}

Figure 1 shows the number of SLR observations (normal points) in the weekly LAGEOS-1/2 solutions, and in the Starlette, Stella, AJISAI solutions. On the average, there are

\footnotetext{
${ }_{3} \mathrm{ftp} / / /$ cddis.gsfc.nasa.gov/pub/slr/products/resource/SLRF2008_ 110913.txt

${ }^{4}$ http://ilrs.dgfi.badw.de/data_handling/ILRS_Data_Handling_File. snx.
} 


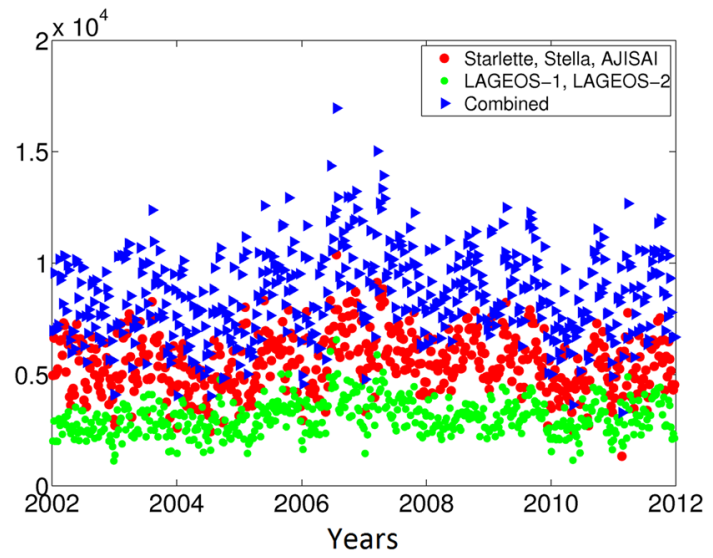

Fig. 1 Number of observations per week in LEO, LAGEOS, and combined solutions

5,500 observations to the LEO satellites and 3,060 observations to the LAGEOS-1/2 satellites contributing to 7-day solutions after screening. Therefore, in a combined solution, the number of observations $(8,560)$ is almost three times larger than the number of SLR observations to the LAGEOS$1 / 2$ satellites only. The largest number of observations was collected in 2006-2007, partly due to a high productivity of the Zimmerwald observatory, where two wavelengths were used simultaneously (Gurtner et al. 2006). Apart from this period, the number of observations is similar for all years, with a characteristic annual signal and minima in winters in the Northern hemisphere.

Most LEO observations are made to AJISAI (3,010 per week) and only 1,677 to Starlette, and eventually 813 to Stella.

The number of parameters is larger in the LEO solution than in the LAGEOS solutions due to the advanced orbit parameterization and due to the estimation of range biases for all stations. The number of estimated parameters is 150,540 , and 580, respectively in the LAGEOS-1/2, the LEO, and the combined solutions. The difference of the number of parameters between the LEO and combined solutions is only 40 , because only LAGEOS orbit parameters and LAGEOS range biases are added, whereas all other parameters are common in both solutions.

Figure 2 shows the number of station contributing to 7day solutions. On an average, 19.8, 21.1, and 22.4 stations contributed to the LAGEOS-1/2, LEO, and the combined solutions, respectively. Therefore, in the combined solution, there are about $10 \%$ more SLR stations than in the LAGEOSonly solution. Consequently, more stations may be used to define the reference frame.

\section{Orbit improvement}

We study different orbit parameterizations of LEO satellites to find a solution that is maximally consistent with external

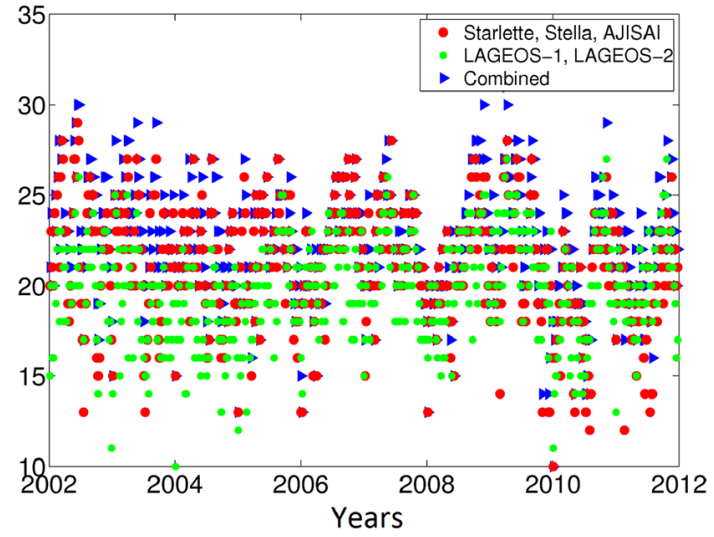

Fig. 2 Number of SLR stations in weekly LEO, LAGEOS, and combined solutions

sources (IERS-08-C04, SLRF2008) using a minimum number of estimated dynamical and stochastic orbit parameters. Moreover, we try to keep the LEO solutions to the greatest extent consistent with the official ILRS ACs' LAGEOSEtalon products.

We investigate the impact of the orbit parameterization on the basic products that are routinely derived by the ILRS, i.e., ERP and station coordinates. In particular, we compare:

- RMS of observation residuals,

- Coordinates of the polar motion and LoD estimates w.r.t. IERS-08-C04 (Petit and Luzum 2011),

- Station coordinates (RMS of 7-parameter Helmert transformation w.r.t. SLRF2008).

\subsection{Parameter interval spacing}

We compare our standard LEO 7-day solution (A, see Table 3), described in Sect. 2 with the solutions characterized by:

- Estimating 1 instead of 7 sets of dynamical parameters per 7-day arc (B1), or estimating 7 sets of dynamical parameters and osculating elements per 7-day solution (B2),

- Estimating pseudo-stochastic pulses in all directions (C1) or neglecting the estimation of pseudo-stochastic pulses (C2),

- Estimating the 6-day solutions with 1 set of osculating elements and 2 sets (D1) or 3 sets (D2) or 6 sets (D3) of dynamical parameters.

All long-arc solutions are estimated by stacking 1-day consecutive normal equations containing all parameters. An approach described by Beutler et al. (1996) is applied for stacking osculating elements, dynamical parameters, and introducing pseudo-stochastic pulses at orbit boundaries. 
Table 3 Impact of different orbit parameterizations of LEO satellites on RMS of observation residuals and ERP (comparison w.r.t. IERS-08-C04)

\begin{tabular}{|c|c|c|c|c|c|c|c|c|c|c|c|}
\hline Solution & $\begin{array}{l}\text { Length } \\
\text { of sol. } \\
\text { (days) }\end{array}$ & $\begin{array}{l}\text { Sets of } \\
\text { oscul. } \\
\text { elem. }\end{array}$ & $\begin{array}{l}\text { Sets of } \\
\text { dynam. } \\
\text { param. }\end{array}$ & $\begin{array}{l}\text { Stoch. } \\
\text { pulses }\end{array}$ & $\begin{array}{l}\text { A posteriori } \\
\text { RMS of } \\
\text { residuals (mm) }\end{array}$ & $\begin{array}{l}X \text { pole } \\
\text { offset } \\
(\mu \text { as })\end{array}$ & $\begin{array}{l}X \text { pole } \\
\text { WRMS } \\
\text { ( } \mu \text { as })\end{array}$ & $\begin{array}{l}Y \text { pole } \\
\text { offset } \\
(\mu \text { as })\end{array}$ & $\begin{array}{l}Y \text { pole } \\
\text { WRMS } \\
\text { ( } \mu \text { as })\end{array}$ & $\begin{array}{l}\text { LoD } \\
\text { offset } \\
(\mu \mathrm{s})\end{array}$ & $\begin{array}{l}\text { LoD } \\
\text { WRMS } \\
(\mu \mathrm{s})\end{array}$ \\
\hline A & 7 & 1 & 7 & $S$ & 7.78 & 57.7 & 269.8 & -8.7 & 218.1 & -3.6 & 106.5 \\
\hline B1 & 7 & 1 & 1 & $S$ & 13.50 & 38.6 & 508.7 & -6.8 & 442.3 & -15.0 & 102.2 \\
\hline B2 & 7 & 7 & 7 & $S$ & 13.42 & 20.7 & 395.7 & 4.4 & 400.1 & -2.2 & 120.0 \\
\hline $\mathrm{C} 1$ & 7 & 1 & 7 & $S, R, W$ & 7.52 & 57.7 & 269.8 & -8.7 & 218.1 & -3.7 & 116.5 \\
\hline $\mathrm{C} 2$ & 7 & 1 & 7 & - & 7.81 & 85.5 & 350.2 & 0.1 & 275.7 & -36.3 & 140.4 \\
\hline D1 & 6 & 1 & 2 & $S$ & 8.21 & 25.7 & 282.6 & 2.4 & 254.2 & -25.4 & 119.7 \\
\hline D2 & 6 & 1 & 3 & $S$ & 7.98 & 28.2 & 280.7 & 10.5 & 244.8 & -13.5 & 115.1 \\
\hline D3 & 6 & 1 & 6 & $S$ & 7.65 & 32.1 & 270.5 & -4.3 & 217.9 & -6.7 & 105.8 \\
\hline
\end{tabular}

The a posteriori errors of ERP from individual solutions are used for estimation of mean offsets and weighted root mean square error (WRMS)

Table 3 gives an overview of orbit parameterization and its impact on ERP estimation, whereas the results of the coordinate comparisons are provided in Fig. 3. Solutions B1 and B2 show a clear degradation of WRMS of pole coordinates, reaching even $100 \%$ for the $X$ pole coordinate WRMS for B1 w.r.t. solution A. Only the WRMS of LoD is slightly improved in solution B1 w.r.t. A, because the daily LoD estimates can be decorrelated better when the dynamical parameters are estimated on a weekly basis. The RMS of the Helmert transformation of station coordinates shows a serious degradation of solutions B1 and B2 w.r.t. the solution A (see Fig. 3). The station coordinates become particularly unstable in B2 when osculating elements are estimated on a daily basis.

The results from solution $\mathrm{C} 1$ are very close to these of solution A. i.e., no further improvement is achieved when estimating stochastic pulses in $W$ and $R$ directions. The estimated values of the parameters are far below their formal a posteriori errors, i.e., they are insignificant. Therefore, the estimation of pulses in $W$ and $R$ can be skipped. Introducing stochastic pulses in all directions may degrade other parameters, e.g., LoD.

Neglecting the estimation of stochastic pulses in $S$ (sol. C2) increases the WRMS of the $X$ pole and the $Y$ pole coordinate by 30 and $26 \%$, respectively (see Table 3 ). The station coordinates are also worse defined in the sol. $\mathrm{C} 2$ than in the sol. A (on an average by $1.4 \mathrm{~mm}$ ). The values of estimated stochastic pulses only in the $S$ direction exceed the value of a posteriori error, i.e., they are significant. We conclude that the stochastic pulses in along-track significantly improve the estimation of ERP and station coordinates.
Fig. 3 RMS of Hemert transformation of SLR-LEO solutions with different orbit modeling w.r.t. SLRF2008 (in $\mathrm{mm})$

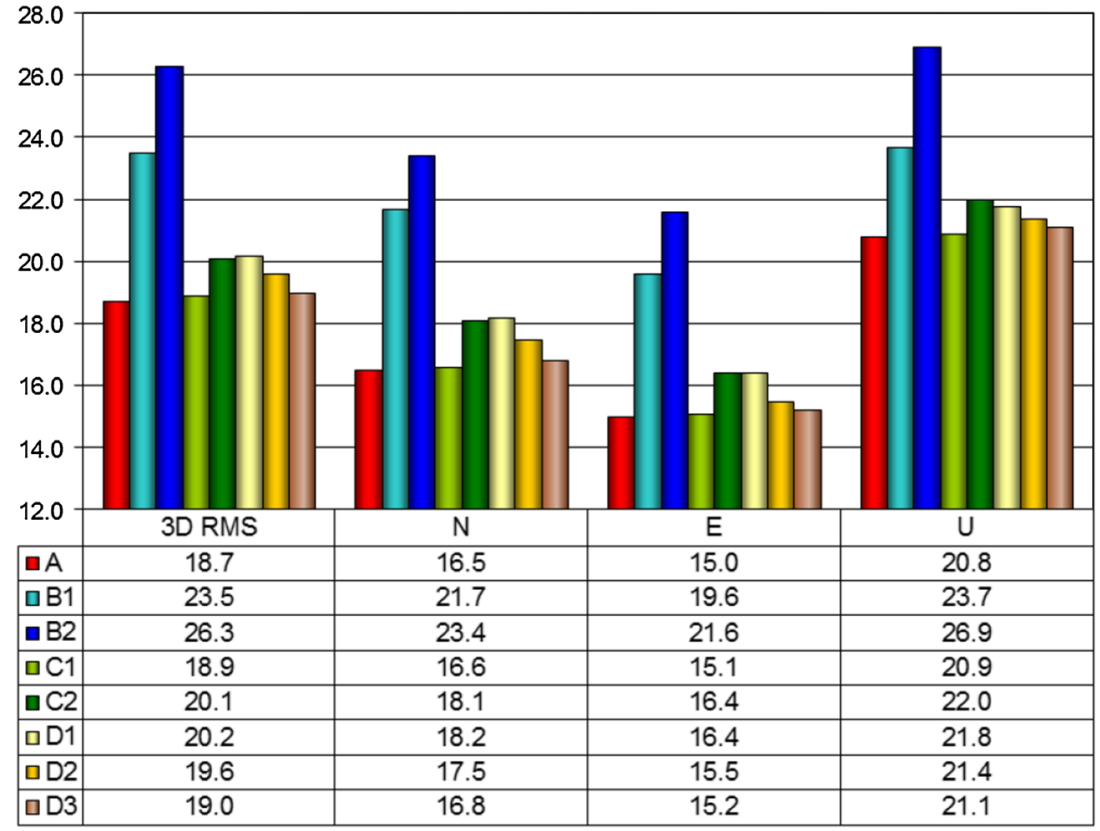


The estimation of the osculating elements, dynamical parameters, and ERP with different parameter spacing (D1, D2) shows a slightly inferior quality w.r.t. solution D3, despite a smaller correlation between the derived parameters.

We selected solution A as the standard LEO solution for subsequent investigations for the sake of consistency with the LAGEOS 7-day solutions and because this solution delivers the best results for most of the estimated parameters.

\subsection{Individual solutions}

Table 4 and Figure 4 illustrate the results from 10 years single-satellite solutions, combined solutions of two satellites, and the combined solution of all LEO satellites (Solution A in Table 3 and Fig. 3). The RMS of observation residuals should not be considered as a conclusive quality indication of solutions, because the degree of freedom strongly differs in the solutions.
The Stella-only solution shows a poor quality for station coordinates and polar motion w.r.t. Starlette-only and AJISAI-only solutions. The WRMS of the $X$ and the $Y$ pole coordinates $(\sim 850 \mu$ as $)$ is about three times larger than for the other single-satellite solutions ( $\sim 250 \mu$ as $)$. There are two reasons for the inferior quality of the Stella solutions: on one hand the small number of observations, on the other hand the specific sun-synchronous orbit and the orbital inclination of $98.6^{\circ}$ resulting in a low sensitivity for polar motion and significant resonances between the apparent diurnal $\left(S_{1}\right)$ and semidiurnal $\left(S_{2}\right)$ motion of the Sun and the Stella's orbit (Cheng et al. 1997).

Station coordinates and polar motion are very well established in the combined AJISAI-Starlette solution, resulting in a 3D RMS of the Helmert transformation of $18.6 \mathrm{~mm}$ and in a WRMS of 207 and $184 \mu$ as for the $X$ pole and $Y$ pole coordinate, respectively. The combined solutions using AJISAI-Stella or Starlette-Stella show a serious degrada-

Table 4 Impact of individual LEO satellite solutions on RMS of observation residuals and ERP (comparison w.r.t. IERS-08-C04)

\begin{tabular}{lllllrrrr}
\hline Solution & $\begin{array}{l}\text { RMS of } \\
\text { observation } \\
\text { residuals }(\mathrm{mm})\end{array}$ & $\begin{array}{l}\text { Mean } \\
\text { number } \\
\text { of observ. }\end{array}$ & $\begin{array}{l}X \text { pole } \\
\text { offset } \\
(\mu \mathrm{as})\end{array}$ & $\begin{array}{l}X \text { pole } \\
\text { WRMS } \\
(\mu \mathrm{as})\end{array}$ & $\begin{array}{l}Y \text { pole } \\
\text { offset } \\
(\mu \text { as })\end{array}$ & $\begin{array}{l}Y \text { pole } \\
\text { WRMS } \\
(\mu \mathrm{as})\end{array}$ & $\begin{array}{l}\text { LoD } \\
\text { offset } \\
(\mu \mathrm{s})\end{array}$ & $\begin{array}{l}\text { LoD } \\
\text { WRMS } \\
(\mu \mathrm{s})\end{array}$ \\
\hline AJISAI-only & 6.31 & 3,011 & 36.4 & 266.3 & 3.6 & 233.9 & -17.3 & 108.5 \\
Starlette-only & 6.45 & 1,697 & 21.8 & 339.5 & -6.5 & 290.5 & -18.0 & 133.0 \\
Stella-only & 6.03 & 813 & 120.0 & 901.6 & -11.8 & 829.0 & 9.6 & 110.7 \\
AJISAI+Starlette & 6.85 & 4,708 & 32.0 & 207.3 & -3.0 & 184.4 & -35.2 & 136.9 \\
AJISAI+Stella & 7.24 & 3,824 & 71.8 & 304.4 & -3.8 & 256.6 & -1.0 & 93.1 \\
Starlette+Stella & 7.62 & 2,510 & 75.2 & 365.2 & -19.1 & 291.5 & -3.7 & 99.4 \\
AJISAI+Starlette+Stella & 7.78 & 5,521 & 57.7 & 269.8 & -8.7 & 218.1 & -3.6 & 106.5 \\
\hline
\end{tabular}

Fig. 4 RMS of Hemert transformation of individual SLR-LEO solutions w.r.t. SLRF2008 (in mm)

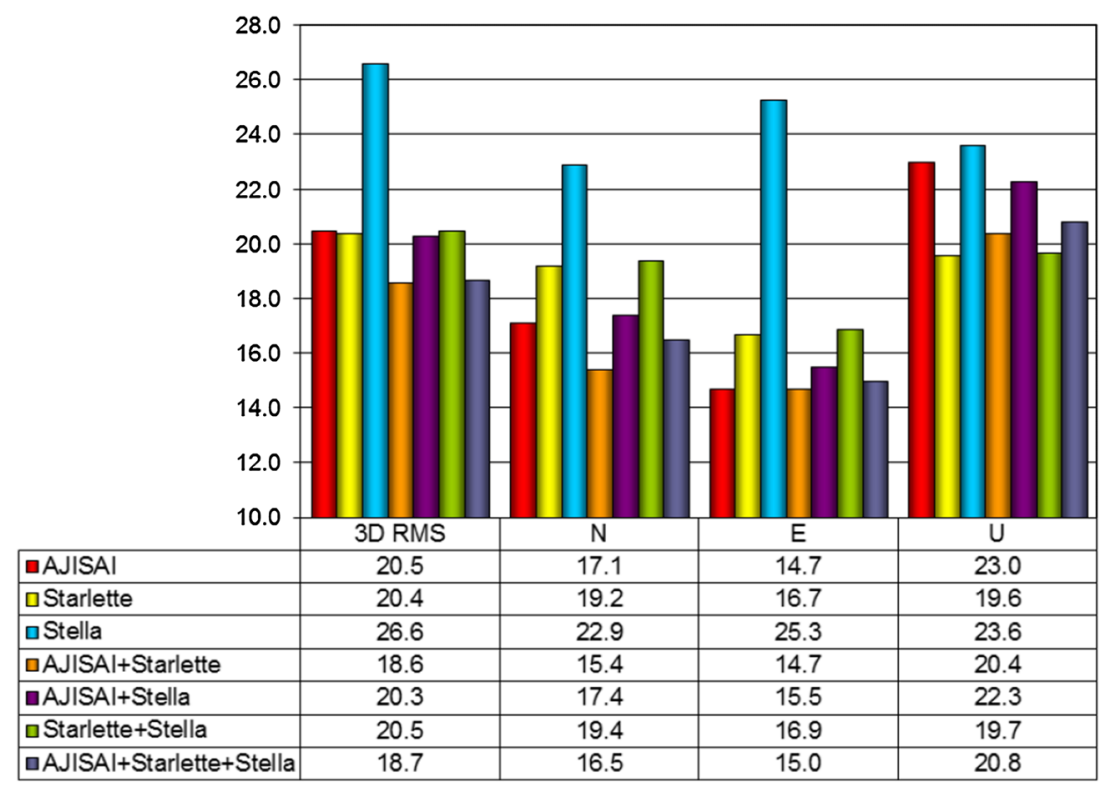


tion of the WRMS of polar motion (up to $365 \mu$ as) and station coordinates (up to $20.5 \mathrm{~mm}$ of 3D RMS). It seems that resonant accelerations influencing Stella's orbit degrade all the tandem solutions with Stella. On the other hand, the offset of LoD and the WRMS of LoD are significantly better: the offset of LoD is reduced from $-35.2 \mu \mathrm{s}$ in the AJISAI-Starlette solution to $-3.7 \mu$ s in the Starlette-Stella and to $-1.0 \mu \mathrm{s}$ in the AJISAI-Stella solutions. The offset of LoD is mostly due to the correlation between $C_{20}$, the dynamic parameter WS, and LoD, because all these parameters stem mostly from the analysis of the drift of ascending node (Thaller et al. 2014b). The drift of satellite's ascending node depends on the orbital inclination, so the decorrelation of $\mathrm{LoD}$ and $C_{20}$ is not possible for two satellites with almost identical inclination angles $\left(50.0^{\circ}\right.$ and $49.8^{\circ}$ for AJISAI and Starlette, respectively). $\mathrm{LoD}$ and $C_{20}$ are decorrelated as soon as Stella is included, despite the resonances of Stella's orbit.

In recent studies, the tandem Starlette-Stella has been used for deriving various geodetic parameters, e.g., for determining elastic Earth parameters (Rutkowska and Jagoda 2012) or estimating station coordinates (Lejba and Schillak 2011). From our study, we conclude that the StarletteStella solution is not well suited for estimating station coordinates (3D RMS of $20.5 \mathrm{~mm}$ ) and polar motion. Much better results can be achieved by combining three LEO satellites.

The AJISAI-Starlette-Stella solution shows only a minor degradation of polar motion estimates w.r.t. the AJISAIStarlette solution. On the other hand, the solution reduces the LoD offset by about a factor of ten. Both, the AJISAIStarlette-Stella and the AJISAI-Starlette solutions, show a similar quality for the station coordinates $(18.6-18.7 \mathrm{~mm}$ of 3D RMS), which is better than in all other combinations (above $20 \mathrm{~mm}$ of 3D RMS for the Helmert transformation).

\section{Combined LEO-LAGEOS solutions}

\subsection{Station coordinates}

Some of the SLR stations in the ILRS network are not capable of observing LAGEOS satellites, or the number and quality of LAGEOS observation are not sufficient for deriving station coordinates. In the latter case, such stations are eliminated during the screening process. The only way to estimate reliable positions for such stations is to use observations of LEO satellites.

In our solution, the positions of six SLR stations are estimated exclusively on the basis of LEO observations: Mendeleevo, Russia (1870), Helwan, Egypt (7831), Lhasa (7356) and Bejing-A, China (7357), Cagliari, Italy (7548) and the mobile French Transportable Laser Ranging Station in Burnie, Tasmania (7370). Not all of these stations are included in the official release of ITRF2008, whereas most of the stations are available in the extended SLRF2008 reference frame, except for Mendeleevo. Most of these stations are, however, temporarily observing sites or provide sparse SLR data of low quality.

Figure 5 shows the estimated coordinate time series of Arequipa, Peru (7403) from the LAGEOS-1/2 and LEO solutions. The coordinates are referred to the SLRF2008. The station provides more observations to LEO satellites, thus the number of LEO solutions is larger than the LAGEOS solutions. The continuous long time series of station coordinates for an SLR station like Arequipa is important, because in the eighties and nineties Arequipa was the only SLR station in South America, bearing in mind that only $15 \%$ of all SLR stations are located in the Southern hemisphere.

The differences of station repeatability in LAGEOS and LEO solutions are shown in Fig. 6. Positive values denote a better repeatability of the LAGEOS solutions, negative values of the LEO solutions. The SLR stations are sorted
Fig. 5 Time series of the Arequipa SLR station coordinates w.r.t. SLRF2008 for LEO solutions and LAGEOS-1/2 solutions
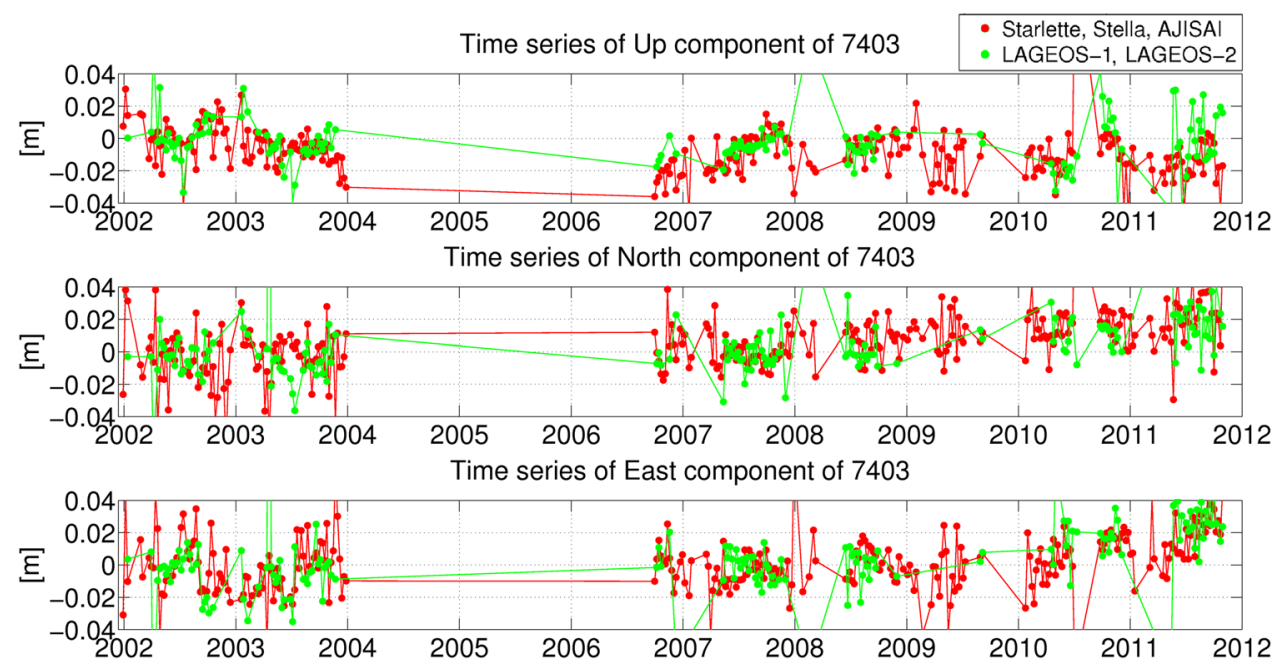
Fig. 6 Difference of the repeatability of station coordinates in the LAGEOS-1/2 solution and the LEO solution. Positive values denote better repeatability in the LAGEOS-1/2 solution. The SLR stations are sorted according to the number of weekly solutions (from the left with the largest number- to the right with the smallest number of weekly solutions)

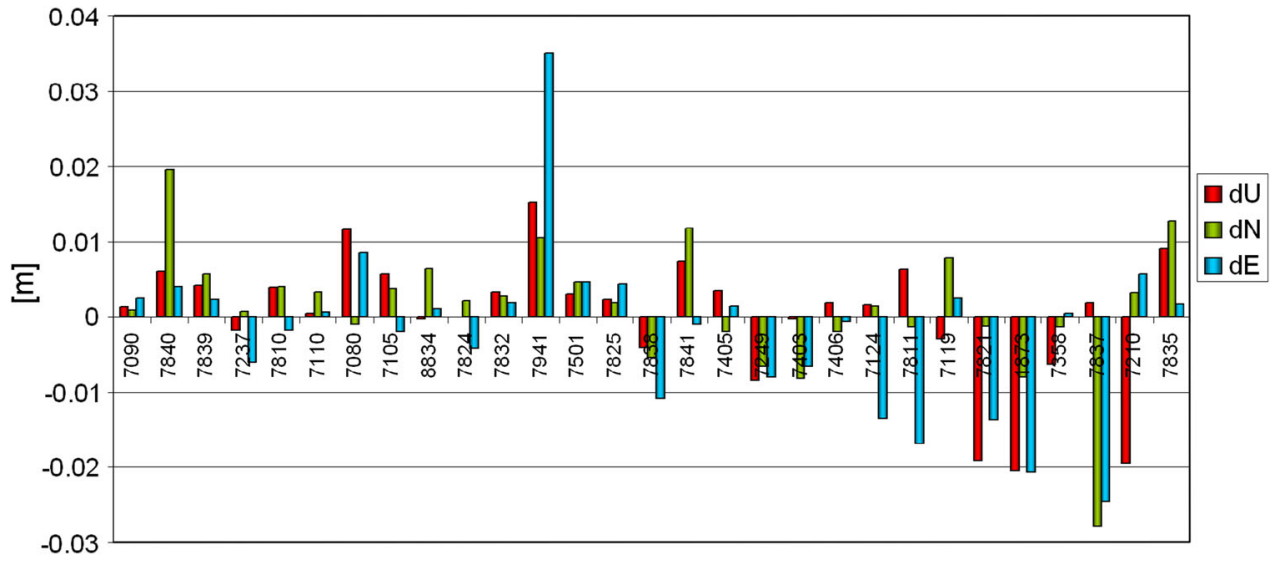

according to the number of weekly solutions. For the high-performing sites, a better repeatability is obtained in the LAGEOS solution. There are a few exceptions, e.g., Changchung, China (7237) and San Fernando, Spain (7824), for which the East component is slightly better determined by the LEO solutions, due to the improved observation geometry. As the range biases are estimated for LEO satellites, we cannot expect a better repeatability of the vertical component in the LEO solutions.

On the other hand, the repeatability of stations contributing to few solutions is better in the LEO solutions, because of the larger number of observations to LEO satellites and because of using three satellites in the LEO solution instead of two satellites in the LAGEOS solution and thus the improved observation geometry. Again, the East component benefits most in LEO solutions w.r.t. LAGEOS solutions, especially for Shanghai, China (7837), Simeiz, Ukraine (1873), Borówiec, Poland (7811), and Tahiti, French Polynesia (7124). The repeatability of other station components is better for stations with a short time span of observations, e.g., for Haleakala, Hawaii (7210) observing for two years within the considered time span.
Figure 7 illustrates the time series of station coordinates for the high-performing core station Zimmerwald. Peaks related to unsatisfactory LEO solutions due to a small number of observations disappear in the combined solution. Before 2008, the vertical component is noisy in all solutions, because range biases are estimated for all satellites, including the LAGEOS satellites. It is remarkable that the vertical component in the combined solution for Zimmerwald is stable, although different wavelengths $(423.0,532.1,846.0 \mathrm{~nm})$ and different SLR receiver systems were used. The major part of equipment changes is, thus, absorbed by range biases, as expected. The vertical component in the LEO solutions is well determined, thanks to a large amount of data collected by Zimmerwald and a good observation geometry, although range biases are estimated to all LEO satellites. Equipment changes show up in the estimated range biases (see Sect. 6).

The difference of the repeatability in the LAGEOS solution and in the combined solution is presented in Fig. 8. Positive values denote a better repeatability in the combined solution, negative values in the LAGEOS solution. In general, the repeatability of station coordinates improves when combining LAGEOS data with the Starlette, Stella, and AJI-
Fig. 7 Time series of the Zimmerwald SLR station coordinates w.r.t. SLRF2008 for LEO, LAGEOS- $1 / 2$, and the combined solutions
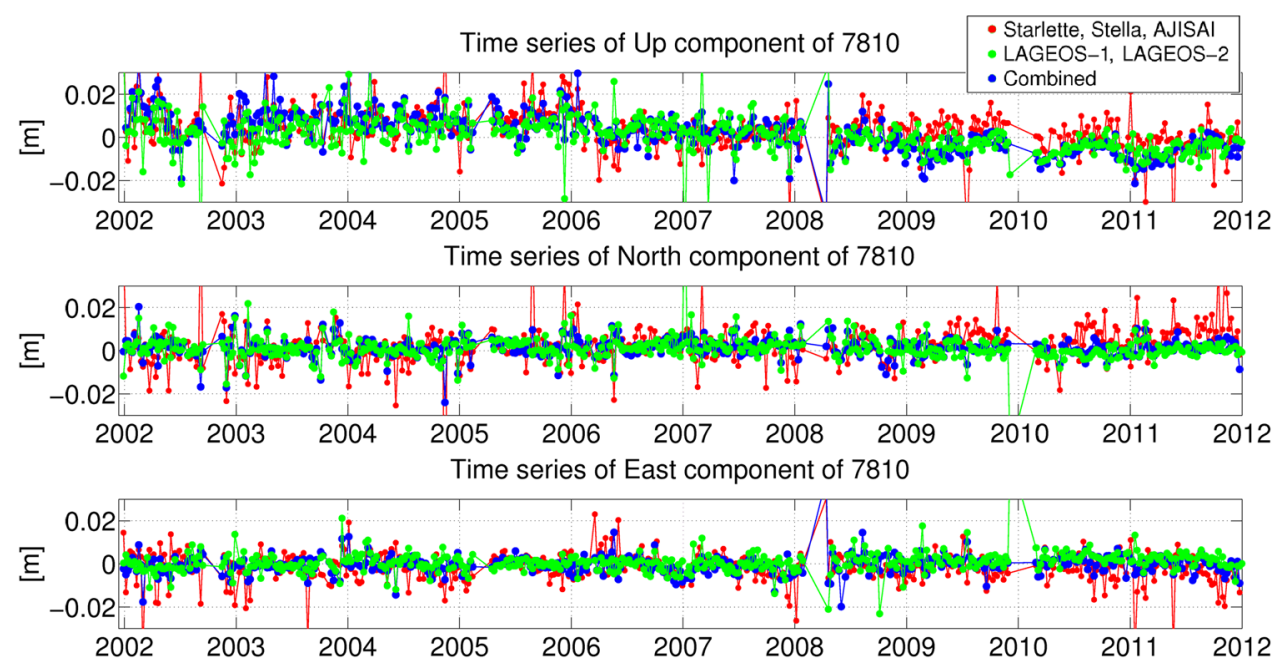
Fig. 8 Difference of the repeatability of station coordinates in the combined solution and in the LAGEOS-1/2 solution. Positive values denote better repeatability in the combined solution

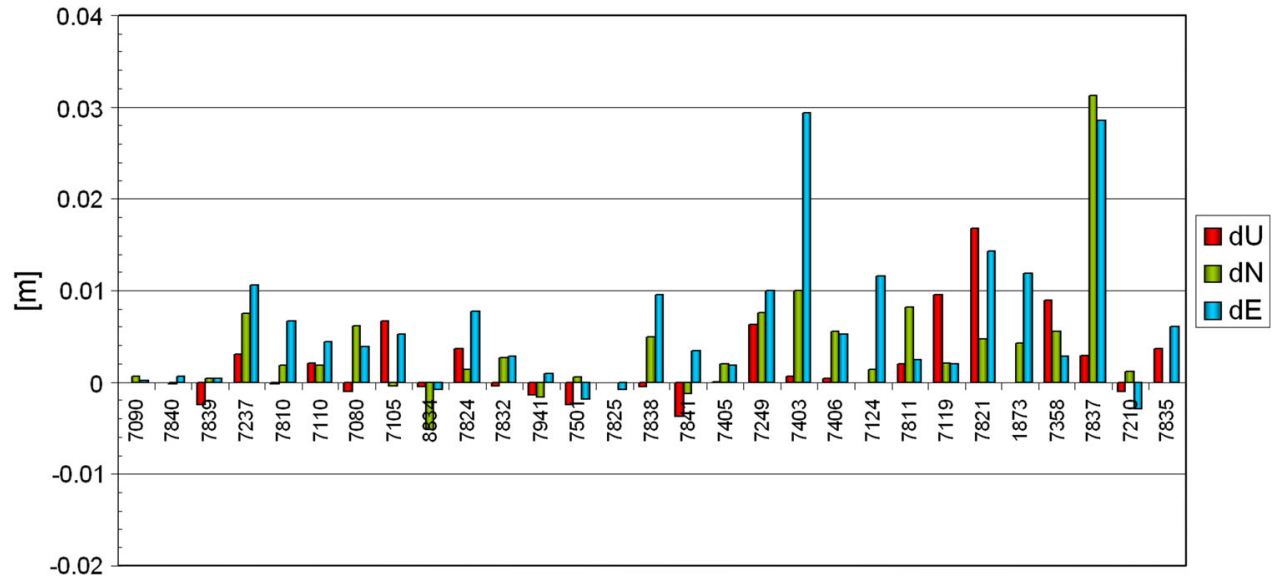

SAI data. The improvement is most prominent for horizontal components and for non-core SLR stations.

We thus conclude that combining LEO with LAGEOS satellites does not worsen the LAGEOS-derived coordinates, but improves in particular the East components of station coordinates due to the mended observation geometry. For some of the SLR stations, coordinates can only be obtained by analyzing the observations to LEO satellites, because, e.g., of the lack of LAGEOS observations.

\subsection{Geocenter}

Figure 9 shows the examples of the $X$ and the $Z$ components of geocenter coordinates resulting from the LEO, LAGEOS, and combined solutions. All series show a similar signal for the geocenter motion, but the LEO solution is noisier than the solutions including the LAGEOS satellites (see Table 5). The mean a posteriori error of the coordinate $X$ of the geocenter $\left(m_{x}\right)$ is smallest for the LAGEOS-only and the combined solutions, amounting 0.74 and $0.72 \mathrm{~mm}$, respectively. For the $Y$ coordinate, a reduction of the a posteriori error is slightly larger, amounting from 0.81 to $0.74 \mathrm{~mm}$, whereas the $Z$ component has the smallest error and shows the smallest scatter in the combined solution $(0.92 \mathrm{~mm}$ in the combined solution, compared to $1.31 \mathrm{~mm}$ in the LAGEOS solution).

The $Z$ component of the geocenter is of special concern, because estimation of this component using satellite techniques is strongly affected by the solar radiation pressure modeling deficiencies (Meindl et al. 2013). Therefore, reliable estimates of the $Z$ geocenter coordinate cannot be derived from GPS, GLONASS, or DORIS satellites.

In the LEO and combined solutions, the errors of the estimation of the amplitude of annual signal in the $Z$ geocenter are smaller than in the LAGEOS solution. The $Z$ compo-
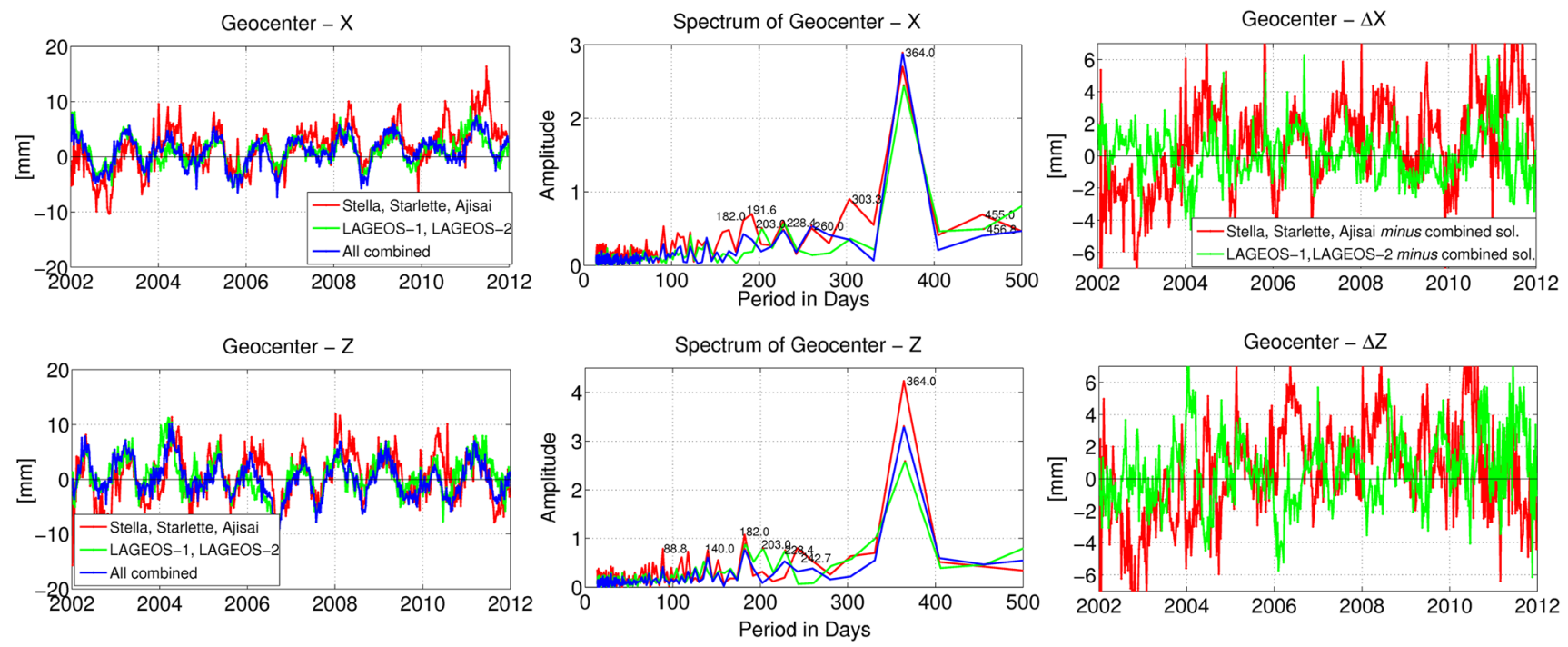

Fig. 9 Time series of the $X$ and the $Z$ components of geocenter coordinates (left), a spectrum analysis (middle) and differences w.r.t. the combined solutions (right). The RMS of differences w.r.t. the combined solutions are $1.5,1.8$, and $2.2 \mathrm{~mm}$ for the $X, Y$, and $Z$ components for the LAGEOS-1/2 solutions, respectively, and 3.1, 3.4, $3.2 \mathrm{~mm}$ for the $X, Y$, and $Z$ components for the Stella, Starlette, AJISAI solution, respectively 
Table 5 Characteristic of geocenter coordinates derived from LAGEOS-1/2, LEO, and combined multi-SLR solutions

\begin{tabular}{|c|c|c|c|c|c|c|c|c|c|}
\hline & \multicolumn{3}{|l|}{$\underline{X}$} & \multicolumn{3}{|l|}{$\underline{Y}$} & \multicolumn{3}{|l|}{$\underline{Z}$} \\
\hline & LEO & LAGEOS & Combined & LEO & LAGEOS & Combined & LEO & LAGEOS & Combined \\
\hline Mean error (mm) & 1.23 & 0.74 & 0.72 & 1.47 & 0.81 & 0.74 & 1.50 & 1.31 & 0.92 \\
\hline Offset & 0.31 & 0.76 & 0.79 & -1.54 & 0.12 & 0.23 & 0.21 & 0.01 & -0.20 \\
\hline$(\mathrm{mm})$ & \pm 0.18 & \pm 0.15 & \pm 0.16 & \pm 0.19 & \pm 0.13 & \pm 0.15 & \pm 0.24 & \pm 0.24 & \pm 0.23 \\
\hline Drift & 0.66 & 0.11 & 0.24 & 1.75 & 0.36 & 0.85 & 0.21 & 0.00 & -0.21 \\
\hline$(\mathrm{mm} / \mathrm{y})$ & \pm 0.06 & \pm 0.05 & \pm 0.05 & \pm 0.06 & \pm 0.04 & \pm 0.04 & \pm 0.07 & \pm 0.07 & \pm 0.07 \\
\hline annual ampl. & 2.97 & 2.99 & 3.40 & 5.28 & 2.49 & 2.94 & 4.68 & 3.64 & 4.13 \\
\hline$(\mathrm{mm})$ & \pm 0.20 & \pm 0.18 & \pm 0.17 & \pm 0.22 & \pm 0.16 & \pm 0.16 & \pm 0.27 & \pm 0.30 & \pm 0.25 \\
\hline semiannual & 2.37 & 0.46 & 1.06 & 0.64 & 0.05 & 0.24 & 1.88 & 1.49 & 1.28 \\
\hline ampl.(mm) & \pm 0.22 & \pm 0.19 & \pm 0.18 & \pm 0.23 & \pm 0.16 & \pm 0.17 & \pm 0.28 & \pm 0.28 & \pm 0.25 \\
\hline
\end{tabular}

Mean offset, drift, amplitudes of annual and semiannual signals are given w.r.t. SLRF2008 and were estimated using the least squares method

nent in the combined solution benefits especially from the Stella orbit, which is almost in a polar orbit. Observations of satellites in polar orbits maximize the topocentric satellite position unit vector along the $Z$ axis of the reference frame (see Eq. 2 from Meindl et al. 2013), which is associated with the maximum of the partial derivatives of the range observations w.r.t. the $Z$ geocenter parameter in the normal equation system. Thus, the satellites in polar or high-inclined orbits carry a valuable information for the determination of the $Z$ geocenter component.

The spectral analysis of the $Z$ coordinate of the geocenter shows that the amplitude associated with the period related to the draconitic year of LAGEOS-2 (the time interval between two subsequent crossings, in the same direction, of the Sun through the satellite's orbital plane, amounting 222 days for LAGEOS-2, see Table 1) is reduced from $0.60 \mathrm{~mm}$ in the LAGEOS-only solution to $0.35 \mathrm{~mm}$ in the combined solution. Therefore, the $Z$ component is even better defined in the combined solution and, as opposed to GNSS estimates, it is less affected by mismodelings of solar radiation pressure (Thaller et al. 2014a). The amplitudes of other signals are also reduced in the $Z$ component, e.g., the semiannual signal from 1.49 to $1.28 \mathrm{~mm}$ (see Table 5).

For all geocenter components, the amplitude of the annual signal is larger in the combined solution than in the LAGEOS solution (see Table 5), on average by $0.45 \mathrm{~mm}$. Low orbiting satellites are more sensitive to the low-degree harmonics of the Earth's gravity field, and thus, they are more sensitive to the variations of the geocenter.

Following Meindl et al. (2013), the decomposition of the accelerations caused by the gravity field coefficient $C_{10}$ into the $R, S, W$ system reads as:

$$
\left(\begin{array}{c}
R \\
S \\
W
\end{array}\right)=C_{10} \frac{G M a_{e}}{r^{3}}\left(\begin{array}{c}
-2 \sin i \sin u \\
\sin i \cos u \\
\cos i
\end{array}\right)
$$

where $C_{10}$ is related to the $Z$ geocenter coordinate, $G M$ is gravitational constant times Earth mass, $a_{e}$ is Earth radius, $r$ is length of the satellite state vector, $i$ is inclination angle, $u$ is argument of latitude of a satellite.

Equation 1 implies that the estimated OPR cosine parameter in the along-track direction (SC) may absorb some geocenter variations, because of the direct correlation with the geocenter-induced perturbing acceleration. Indeed, the time series of the SC parameter in LAGEOS-1/2 solutions includes two main signals: one corresponding to the draconitic years of LAGEOS, and second corresponding to the annual signal. The presence of the draconitic year signal implies that the SC parameter, as expected, absorbs some non-gravitational satellite accelerations related to the direct or indirect solar radiation pressure, whereas the annual signal can be associated with some geophysical phenomena, e.g., the geocenter motion. The correlation coefficients between the $Z$ geocenter coordinate and the SC parameter are, e.g., - 0.83, and 0.58 for LAGEOS-1, and LAGEOS-2, respectively in a 7 -day LAGEOS-1/2 solution. These correlations are reduced to -0.23 and 0.15 in the multi-SLR solutions. Thus, we conclude that in the LAGEOS-only solutions with the estimation of the standard set of empirical parameters (including SC, see Table 2), some of the geocenter signals are absorbed by the empirical orbit parameters. In particular, the amplitude of annual signal is underestimated for the $Z$ geocenter coordinate in the LAGEOS$1 / 2$ solutions. In the multi-SLR solutions, the correlation coefficients between the empirical parameters and the geocenter coordinates are substantially reduced, whereas the amplitudes of the annual signal are increased. Finally, the influence of the solar radiation pressure, which is related to the orbit perturbations of the draconitic year signal, is absorbed by the empirical orbit parameters to the greatest extent in the multi-SLR solutions. Thus, the geocenter coordinates are less contaminated by orbit modeling deficiencies. 
The amplitudes of other than annual and semiannual signals in $X$ and $Y$ coordinates of the geocenter can slightly be increased in the combined solution, but none of these amplitudes exceed the value of $0.5 \mathrm{~mm}$. In summary, the quality of the estimated geocenter coordinates is comparable for the $X$ and $Y$ components in the LAGEOS-1/2 and in the combined solutions, whereas the quality of the $Z$ coordinate is better in the combined multi-SLR solutions.

\subsection{Earth rotation parameters}

Table 6 shows the mean offsets and WRMS values of the pole coordinates and LoD estimates w.r.t. the a priori IERS08-C04 series (Bizouard and Gambis 2012). In all cases, the mean offsets are not significant and they agree very well with the IERS-08-C04 series. The pole coordinates from the LEO solutions are worse in terms of the weighted RMS by a factor 1.6 than the estimates derived from the LAGEOS solutions.

Schutz et al. (1989) reported that the best obtained pole coordinates from Starlette data agreed within 4.4 and 3.6 mas for the $X$ pole and the $Y$ pole, respectively, with values obtained from LAGEOS-1 observations. The agreement of pole coordinates between LEO and LAGEOS solutions in this analysis is at the level of 0.2 mas. The quality of SLR-derived ERP has obviously been dramatically improved within the time span of 20 years. The improvement is mostly due to using a larger number of satellites, a denser network of SLR stations, improved background models, and substantially better quality of SLR data.

The WRMS of the pole coordinates is reduced from 160 and $155 \mu$ as in the LAGEOS solution for the $X$ and $Y$ coordinates of polar motion, respectively, to 149 and $140 \mu$ as in the combined solution (see Table 6; Fig. 10). This corresponds to a reduction of 7 and $10 \%$, respectively. The differences in LoD estimates between LAGEOS and the combined solution are almost negligible.

As shown in Sect. 4.2, the incorporation of Stella degrades the WRMS of polar motion in a LEO solution. Therefore, we generated another multi-satellite solution by excluding Stella. The solution did, however, not show any difference of

Table 6 Pole coordinates and Length-of-day estimates w.r.t. a priori IERS-08-C04 series

\begin{tabular}{llrrr}
\hline & & LEO & LAGEOS & Combined \\
\hline \multirow{4}{*}{ Mean offset } & $X$ pole $(\mu$ as $)$ & 57.7 & 4.1 & 6.4 \\
& $Y$ pole $(\mu$ as $)$ & -8.7 & -8.0 & -8.5 \\
& LoD $(\mu \mathrm{s})$ & -3.6 & 6.1 & 6.3 \\
\multirow{4}{*}{ WRMS } & $X$ pole $(\mu$ as $)$ & 269.8 & 160.0 & 148.9 \\
& $Y$ pole $(\mu$ as $)$ & 218.1 & 155.2 & 140.3 \\
& LoD $(\mu \mathrm{s})$ & 106.5 & 57.0 & 56.3 \\
\hline
\end{tabular}
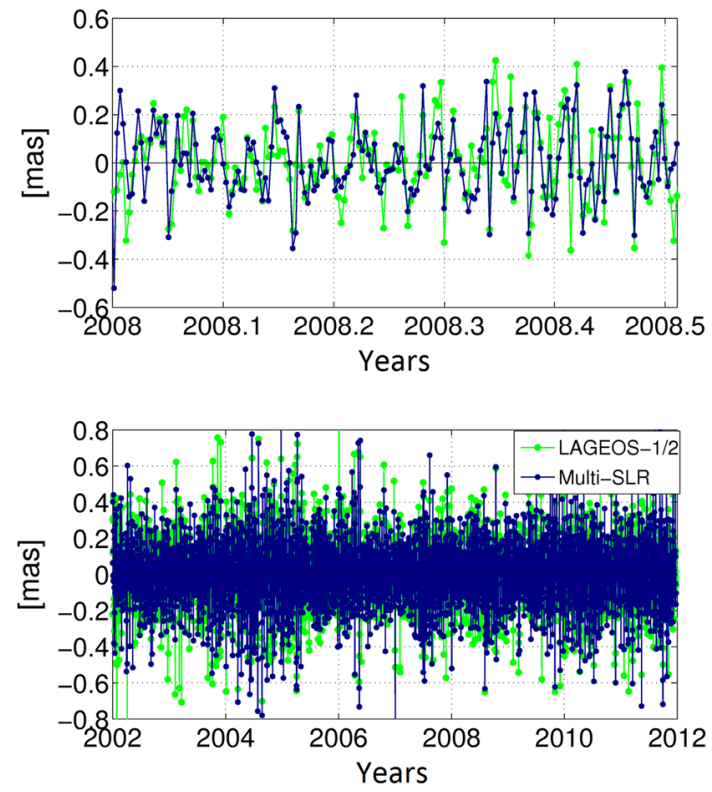

Fig. $10 X$ pole coordinates w.r.t. IERS-08-C04 series for half a year (top) and entire time series (bottom)

the polar motion and LoD w.r.t. the solution with five satellites, because of the heavy impact of the LAGEOS satellites.

\subsection{Scale}

Figure 11 shows the weekly scale estimates from the Helmert transformation w.r.t. the a priori reference frame SLRF2008. In the LAGEOS-1/2 solutions, the scale is well established,
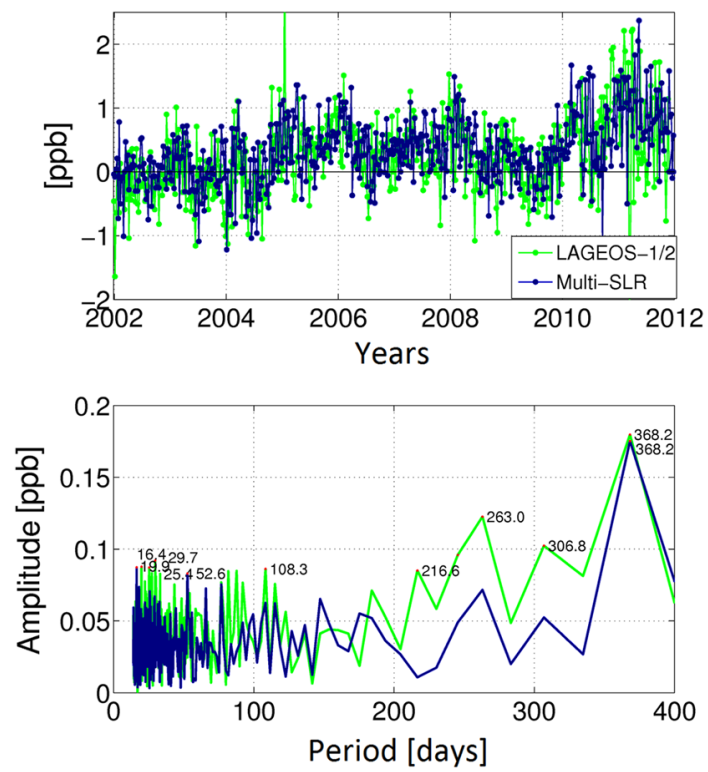

Fig. 11 Time series of scale estimates from Helmert transformation w.r.t. SLRF2008 (top) and the amplitude spectrum of scale differences (bottom) 
because no range biases to the LAGEOS satellites have to be estimated for most of the SLR stations. Therefore, the scale in the combined solutions is defined almost uniquely by LAGEOS, because station- and satellite-specific range biases to the LEO satellites absorb the discrepancies in the scale estimates.

Both scale estimates agree to within $1 \mathrm{ppb}$ for most of the epochs. The mean values of scale differences w.r.t. SLRF2008 are 0.24 and 0.33 ppb for the LAGEOS and the combined solutions, respectively. These values correspond to 1.5 and $2.1 \mathrm{~mm}$. The scale differences in the LAGEOS and in the combined solutions are not significant statistically from the SLRF2008 scale, because the WRMS of the scale differences are 0.58 and $0.57 \mathrm{ppb}$, respectively. Moreover, the discrepancy between the LAGEOS and multi-SLR scales is one order of magnitude smaller than the discrepancy between VLBI and SLR scales in ITRF2008 (Altamimi et al. 2011)

The amplitude spectrum of the combined solution shows similar amplitudes of the annual signal for the LAGEOS and the combined solutions. Artifactual spectral lines from the LAGEOS solution (caused by the mismodelings of satellite orbits) are reduced, e.g., the peak of 216.6 days, corresponding to the draconitic year of LAGEOS-2, the 108.3 day period and the 263.0 day period corresponding to the eclipsing periods of LAGEOS-2 and LAGEOS-1, respectively, and the 306.8 day period corresponding to a revolution period of equatorial longitude of LAGEOS-2 perigee w.r.t. the Sun. In the periodogram of the combined solution, only three amplitudes exceed the level of $0.08 \mathrm{ppb}$, corresponding to the annual signal, the 16.4 days, and the 52.6 days. In the LAGEOS solution, 13 amplitudes exceed the threshold of $0.08 \mathrm{ppb}$.

\subsection{Correlations}

Figure 12 illustrates the correlation matrices derived from the reduced normal equation systems of the LAGEOS-1/2 solution and of the multi-SLR solution (one weekly solution in December 2011). The matrices contain the station coordinates (parameters 1-36), ERP (37-60), and the Earth gravity field parameters (61-75). All remaining parameters were pre-eliminated, and thus, they are implicitly included in the correlation matrices.

The maximum correlation between the Earth gravity field parameters and station coordinates is reduced from 0.53 in the LAGEOS-1/2 solution to 0.18 in the multi-SLR solution. The correlations between pole coordinates and UT1UTC are also greatly reduced in the multi-SLR solution. The only correlations between the ERP in the multi-SLR solution exceeding 0.25 are those between UT1-UTC for consecutive days, which is not surprising, because the PWL parameterization is applied with constraining only one UT1-UTC parameter. Finally, the correlations between station coordinates are also reduced due to the larger amount of observations and a much better observation geometry.

\section{Range biases}

The satellite's orbit refers to the satellite center-of-mass, i.e., the point whose motion reflects the orbital dynamics. The laser impulse is reflected by retroreflectors embedded on the surface of the spherical satellite. This is why a CoM correction is needed to extrapolate the laser range measurements to the satellite's center-of-mass. The value of CoM correction depends on the size, shape, type, and properties of the corner cubes, as well as properties of the ground-based systems, i.e., pulse energy, pulse width, wavelength, and receiver characteristics (Otsubo and Appleby 2003).

We estimate the satellite- and station-specific differential range biases $(\triangle \mathrm{RB})$ to account for station-specific CoM corrections and some other station-specific systematic biases. $\triangle \mathrm{RB}$ can be transformed to the CoM corrections, but then the estimated CoM corrections contain not only the differences between reflecting points and the center-of-mass of the satellites, but also the properties of the ground-based systems and other residual biases $\left(\epsilon_{\text {Bias }}\right)$. $\Delta \mathrm{RB}$ are derived w.r.t. a priori range biases from the ILRS data handling file, provided
Fig. 12 Correlation matrix for LAGEOS- $1 / 2$ solution and multi-SLR solution
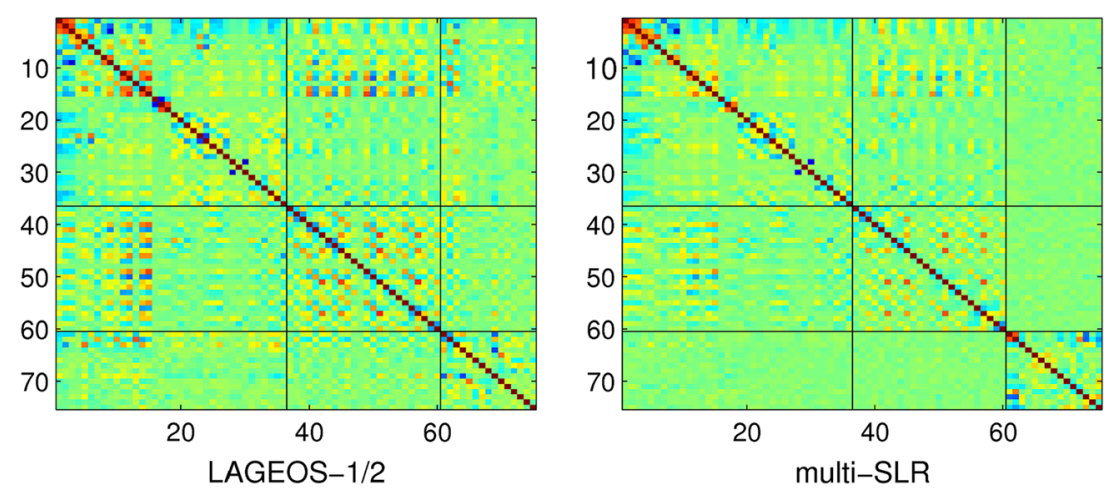

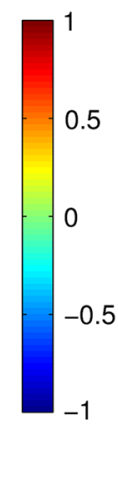


Table 7 Impact of the a priori CoM and estimation of range biases

\begin{tabular}{|c|c|c|c|c|c|c|c|c|c|c|c|}
\hline \multirow{2}{*}{$\begin{array}{l}\text { Range } \\
\text { biases }\end{array}$} & \multirow[t]{2}{*}{$\mathrm{CoM} / \Delta \mathrm{RB}$} & \multirow{2}{*}{$\begin{array}{l}\text { RMS of } \\
\text { resid. } \\
(\mathrm{mm})\end{array}$} & \multirow{2}{*}{$\begin{array}{l}X \text { pole } \\
\text { offset } \\
(\mu \text { as })\end{array}$} & \multirow{2}{*}{$\begin{array}{l}X \text { pole } \\
\text { WRMS } \\
(\mu \text { as })\end{array}$} & \multirow{2}{*}{$\begin{array}{l}Y \text { pole } \\
\text { offset } \\
(\mu \text { as })\end{array}$} & \multirow{2}{*}{$\begin{array}{l}Y \text { pole } \\
\text { WRMS } \\
(\mu \text { as })\end{array}$} & \multirow{2}{*}{$\begin{array}{l}\text { LoD } \\
\text { offset } \\
(\mu \mathrm{s})\end{array}$} & \multirow{2}{*}{$\begin{array}{l}\text { LoD } \\
\text { WRMS } \\
(\mu \mathrm{s})\end{array}$} & \multicolumn{3}{|c|}{ RMS of Helmert } \\
\hline & & & & & & & & & $\begin{array}{l}\mathrm{U} \\
(\mathrm{mm})\end{array}$ & $\begin{array}{l}\mathrm{N} \\
(\mathrm{mm})\end{array}$ & $\begin{array}{l}\mathrm{E} \\
(\mathrm{mm})\end{array}$ \\
\hline Fixed & Standard $^{1}$ & 7.96 & 240.8 & 352.2 & -81.0 & 322.8 & -5.1 & 118.2 & 26.3 & 19.3 & 16.8 \\
\hline Estimated & Standard ${ }^{1}$ & 7.78 & 57.7 & 269.8 & -8.7 & 218.1 & -3.6 & 106.5 & 20.8 & 16.5 & 15.0 \\
\hline Estimated & Re-substituted ${ }^{2}$ & 7.78 & 57.7 & 269.7 & -8.7 & 218.0 & -3.6 & 106.6 & 20.7 & 16.5 & 15.0 \\
\hline Fixed & Re-substituted ${ }^{2}$ & 7.84 & 38.3 & 267.9 & -7.8 & 217.6 & -3.8 & 105.6 & 18.5 & 16.1 & 14.9 \\
\hline
\end{tabular}

Comparison w.r.t. the IERS-08-C04 series for polar motion and w.r.t. SLRF2008 for the Helmert transformation

${ }^{1}$ Standard CoM corrections: $1,100 \mathrm{~mm}$ for AJISAI and $75 \mathrm{~mm}$ for Starlette/Stella. One value for all SLR stations

${ }^{2}$ Empirical station-specific $\triangle \mathrm{RBs}$ re-substituted into the solution

that the largest and well-known biases are a priori removed and not included in the resulting $\triangle \mathrm{RB}$ series.

The $\triangle \mathrm{RB}$ are estimated to all LEO satellites and for all SLR stations. For LAGEOS satellites, $\triangle \mathrm{RB}$ are estimated only for selected periods and stations, following the recommendations from the ILRS data handling file (see Sect. 2.4). For all other stations, the CoM corrections to the LAGEOS satellites are fixed to well-established station- and satellitespecific corrections, as provided by Appleby et al. (2012) ${ }^{5}$. Appleby et al. (2012) generated the CoM corrections for LAGEOS and Etalons using the method of matching full rate residual histograms with theoretical response functions. These new CoM corrections were adopted for generation of official ILRS products starting from October 1, 2013.

We found that $\triangle \mathrm{RB}$ for Starlette and Stella varies between -14 and $+11 \mathrm{~mm}$, but the weighted mean of $\triangle \mathrm{RB}$ for all stations is only $0.2 \mathrm{~mm}$. This corresponds to the mean CoM correction amounting $77.8 \mathrm{~mm}$. This result confirms the statement by Ries (2008) that the standard CoM correction value of $75 \mathrm{~mm}$ is not valid for currently operating SLR systems. Ries (2008) also states that the CoM corrections for Starlette and Stella are between 78 and $79 \mathrm{~mm}$. Otsubo et al. (2012) also claim that the CoM correction for Starlette should be 78$79 \mathrm{~mm}$ for multiphoton systems and 75-79 $\mathrm{mm}$ for singlephoton systems.

For AJISAI, the estimated $\triangle \mathrm{RB}$ is between -6 and $46 \mathrm{~mm}$, with a weighted mean of $16.1 \mathrm{~mm}$. This implies a significant difference between the standard AJISAI CoM value $(1,010 \mathrm{~mm})$ and the empirical CoM correction (993.9 $\mathrm{mm})$, which is based on estimated $\triangle \mathrm{RB}$. Using the standard CoM value for AJISAI (i.e., the same value for all SLR stations) leads to degraded solutions affecting in particular the vertical component of station coordinates (Sośnica et al. 2012b). The wrong a priori values of the CoM corrections also explain the systematic shifts of the vertical component reported by Lejba and Schillak 2011, amounting, e.g., $-33.2 \mathrm{~mm}$ for the Yarragadee station, $-36.8 \mathrm{~mm}$ for Herstmonceux, +19.5 for Graz, and $-28.4 \mathrm{~mm}$ for Greenbelt in the AJISAI-only solu-

\footnotetext{
5 http://ilrs.gsfc.nasa.gov/docs/LAGEOS_CoM_Table_081023.pdf.
}

tion. Lejba and Schillak (2011) used the fixed CoM standard corrections without estimating station-specific range biases. Using station- or system-specific CoM corrections is, thus, necessary for large-size SLR spacecrafts like AJISAI, which was already pointed out by Otsubo and Appleby (2003).

Table 7 shows the results of the LEO solutions with different treatment of range biases and a priori CoM corrections. Fixing the CoM corrections to one standard value causes an offset of up to $240 \mu$ as for the $X$ pole coordinate, corresponding to $10 \mathrm{~mm}$ on the Earth's surface. The offset vanishes when estimating range biases for all SLR stations or using the resubstituted station-specific $\triangle \mathrm{RB}$ values. The estimation of range biases leads to the acceptable solutions even if the a priori CoM corrections are incorrect. Not estimating range biases increases the RMS of residuals in all cases, because the number of parameters is reduced and the degree of freedom of the solution is changed. Nevertheless, solution 4 from Table 7 with fixed range biases and re-substituted stationspecific $\triangle R B$ values shows a further slight improvement of ERP estimates as compared to the solutions with estimating range biases.

The Helmert transformation of station coordinates w.r.t. SLRF2008 shows a degradation of the vertical component when the CoM corrections are fixed to the standard values (on average a repeatability of $26.3 \mathrm{~mm}$ ) as compared to the solution when the $\triangle \mathrm{RBs}$ are fixed to station-specific values (the repeatability of $18.5 \mathrm{~mm}$ ).

\section{Conclusions and recommendations}

We showed that combining the single-satellite Starlette, Stella, and AJISAI NEQs with the LAGEOS-1/2 NEQs strengthens the SLR-derived geodetic parameters. The number of SLR normal points is on average three times larger in combined solutions than in the LAGEOS-only solutions. For some SLR stations, e.g., Mendeleevo in Russia and Helwan in Egypt, the station coordinates can only be obtained from SLR data to LEO satellites, because of an insufficient number or even a complete absence of LAGEOS observa- 
tions. The repeatability of the East and North components of station coordinates is improved in the combined solution for those SLR stations with small numbers of LAGEOS observations.

We found that incorporating Stella into the combined solution using low orbiting spherical satellites is important for the decorrelation of LoD and $C_{20}$, despite Stella's sunsynchronous orbit showing resonances with the diurnal and semidiurnal motion of the Sun. On the other hand, incorporating Stella slightly degrades the station and pole coordinates in the solution using only LEO satellites. Moreover, the tandem Starlette-Stella turned out not to be best suited for the estimation of station coordinates and polar motion. Much better results can be achieved from the AJISAI-Starlette-Stella solution.

The estimation of pseudo-stochastic pulses in the alongtrack direction improves not only the orbits of the LEO satellites, but also other SLR-derived parameters. Solutions without pulses increase the WRMS of the $X$ and the $Y$ coordinate of polar motion by 30 and $26 \%$, respectively. The estimation of pulses in the out-of-plane and in the radial directions does not further improve the solution. In our study, the best results were achieved when forming long arcs of 6-7 days and when setting up empirical dynamic parameters on a daily basis (Solution A and D3 in Table 3).

The $Z$ component of geocenter coordinates is of better quality when combining several geodetic satellites than in pure LAGEOS-1/2 solutions; the mean a posteriori error is decreased (from 1.3 to $0.9 \mathrm{~mm}$ ) and the correlation coefficient between once-per-revolution empirical orbit parameters SC and the $Z$ geocenter coordinate is reduced (e.g., from -0.83 to -0.23 for LAGEOS -1$)$. In a combined solution, the amplitudes of the annual signal for all geocenter components are increased by about $0.45 \mathrm{~mm}$ as compared to the LAGEOS-only solutions, whereas the amplitude of the period related to the draconitic year of LAGEOS-2 is reduced from $0.60 \mathrm{~mm}$ in the LAGEOS-1/2 solutions to $0.35 \mathrm{~mm}$ in the combined multi-SLR solutions.

The WRMS of the pole coordinates w.r.t. IERS-08-C04 series is reduced from 160 and $155 \mu$ as for the $X$ and $Y$ coordinates in the LAGEOS-1/2 solution, respectively, to 149 and $140 \mu$ as for the $X$ and $Y$ coordinates of polar motion in a combined solution. It corresponds to an improvement of $7 \%$ for the $X$ and $10 \%$ for the $Y$ coordinate.

A spectral analysis of the scale of the SLR network w.r.t. SLRF2008 shows that the artifacts related to orbit perturbations in the LAGEOS solutions, e.g., periods related to the draconitic year, are remarkably reduced in the combined solutions.

The parameters derived from the multi-satellite solutions are of better quality compared to the single-satellite solutions. The multi-satellite LAGEOS-1-Starlette- AJISAI solutions should, therefore, be considered for the realization of the reference frame, in particular for the period before the launch of the LAGEOS-2, instead of a realization based only on LAGEOS-1 data.

Finally, we showed that the AJISAI and Starlette/Stella standard Center-of-Mass Corrections, i.e., one value for all SLR stations, are not best suited for the currently operating SLR systems. This fact has already been recognized by the ILRS and is remedied through the adoption of the new, siteand time-dependent CoM models for all targets that contribute to official ILRS products, i.e., LAGEOS and Etalon satellites. The variations of differential range biases reach 52 and $25 \mathrm{~mm}$ for AJISAI and Starlette-Stella, respectively. Therefore, it is recommended that the station-specific CoM corrections be used instead of one value for all SLR stations or the range biases should be estimated for all SLR stations. The estimation of range biases leads to the acceptable solutions even if the a priori CoM corrections are incorrect. The station-specific range biases for AJISAI from this study explain a systematic shift of the vertical component of station coordinates in the AJISAI solutions reported by Lejba and Schillak (2011).

Acknowledgments The ILRS (Pearlman et al. 2002) is acknowledged for providing SLR data. SLR stations are acknowledged for a continuous tracking of geodetic satellites. We acknowledge the ILRS Analysis Working Group for the permanent care of the highest quality of the SLR-derived products.

\section{References}

Altamimi Z, Collilieux X, Métivier L (2011) ITRF2008: an improved solution of the international terrestrial reference frame. J Geod 85(8):457-473. doi:10.1007/s00190-011-0444-4

Appleby G, Otsubo T, Pavlis EC, Luceri C, Sciarretta C (2012) Improvements in systematic effects in satellite laser ranging analysessatellite centre-of-mass corrections. Geophysical Research Abstracts 14, EGU2012-11566, EGU General Assembly

Beutler G, Brockmann E, Gurtner W, Hugentobler U, Mervart L, Rothacher M (1994) Extended orbit modeling techniques at the CODE processing center of the international GPS service for geodynamics (IGS): theory and initial results. Manuscr Geod 19(6):367386

Beutler G, Brockmann E, Hugentobler U, Mervart L, Rothacher M, Weber R (1996) Combining consecutive short arcs into long arcs for precise and efficient GPS orbit determination. J Geod 70(5):287299. doi:10.1007/BF00867349

Beutler G, Jäggi A, Mervart L, Meyer U (2010) The celestial mechanics approach - theoretical foundations. J Geod 84(10):605-624. doi:10. 1007/s00190-010-0401-7

Bizouard C, Gambis D (2012) The combined solution C04 for Earth Orientation parameters consistent with International Terrestrial Reference Frame 2008. Observatoire de Paris, Syrte 61 av. de l'Observatoire Paris, France

Cheng M, Shum C, Eanes J, Schutz B, Tapley B (1990) Long-period perturbations in Starlette orbit and tide solution. J Geophys Res 95(B6):8723-8736. doi:10.1029/JB095iB06p08723

Cheng M, Shum C, Tapley B (1997) Determination of long-term changes in the earth's gravity field from satellite laser ranging observations. J Geophys Res 102(B10):22377-22390. doi:10.1029/ 97JB01740 
Dach R, Hugentobler U, Fridez P, Meindl M (2007) Bernese GPS Software Version 5.0. Astronomical Institute, University of Bern, Switzerland

Dach R, Brockmann E, Schaer S, Beutler G, Meindl M, Prange L, Bock H, Jäggi A, Ostini L (2009) GNSS processing at CODE: status report. J Geod 83(3-4):353-365. doi:10.1007/s00190-008-0281-2

Gourine B (2012) Use of Starlette and LAGEOS-1\&-2 laser measurements for determination and analysis of stations coordinates and EOP time series. C R Geosci 344(6):319-333. doi:10.1016/j.crte. 2012.05.002

Gurtner W, Pop E, Utzinger J (2006) Two-color calibration of The Zimmerwald SLR system. In: Proceedings of the 15th international workshop on laser ranging in Canberra, Australia

Jäggi A, Hugentobler U, Beutler G (2006) Pseudo-stochastic orbit modeling techniques for low-earth orbiters. J Geod 80(1):47-60. doi:10. 1007/s00190-006-0029-9

Jäggi A, Sośnica K, Thaller D, Beutler G (2012) Validation and estimation of low-degree gravity field coefficients using LAGEOS. In: Proceedings of 17th ILRS Workshop, vol 48, Bundesamt für Kartographie und Geodäsie, Frankfurt, ISBN 978-3-89888-999-5

Lejba P, Schillak S (2011) Determination of station positions and velocities from laser ranging observations to Ajisai, Starlette and Stella satellites. Adv Space Res 47(4):654-662. doi:10.1016/j.asr.2010.10. 013 ISSN 0273-1177

Meindl M, Beutler G, Thaller D, Dach R, Jäggi A (2013) Geocenter coordinates estimated from GNSS data as viewed by perturbation theory. Adv Space Res 51(7):1047-1064. doi:10.1016/j.asr.2012.10. 026

Métris G, Vokrouhlicky D, Ries JC, Eanes JR (1997) Nongravitational effects and the LAGEOS eccentricity excitations. J Geophys Res 102(B2):2711-2729. doi:10.1029/96JB03186

Otsubo T, Appleby G (2003) System-dependent center-of-mass correction for spherical geodetic satellites. J Geophys Res 108(B4):2201. doi:10.1029/2002JB002209

Otsubo T, Amagai J, Kunimori H (1999) The center of mass correction of the geodetic satellite AJISAI for single-photon laser ranging. IEEE Trans Geosci Remote Sens 37(4): doi:10.1109/36.774712

Otsubo T, Sherwood R, Appleby G (2012) Target signatures of existing sub-cm targets and prospects for future SLR constellations. In: Proceedings of the international technical laser workshop 2012 (ITLW-12), Frascati (Rome), Italy, http://www.lnf.infn. it/conference/laser2012/2tuesday/3_4_1otsubo/otsubo_l.pdf

Paolozzi A, Ciufolini I (2013) Lares succesfully launched in orbit: satellite and mission description. Acta Astron. ISSN 0094-5765, doi:10. 1016/j.actaastro.2013.05.011

Pavlis NK, Holmes SA, Kenyon SC, Factor JK (2012) The development and evaluation of the earth gravitational model 2008 (EGM2008). J Geophys Res 117(B04):406. doi:10.1029/2011JB008916

Pearlman MR, Degnan JJ, Bosworth JM (2002) The international laser ranging service. Adv Space Res 30(2):135-143. doi:10.1016/ S0273-1177(02)00277-6

Petit G, Luzum B (2011) IERS Conventions 2010. IERS Technical Note 36. Verlag des Bundesamts für Kartographie und Geodäsie, Frankfurt am Main

Picone JM, Hedin AE, Drob DP, Aikin AC (2002) NRL-MSISE00 empirical model of the atmosphere: statistical comparisons and scientific issues. J Geophys Res 107(1468):16. doi:10.1029/ 2002JA009430
Ries J (2008) SLR bias/CoM offset issues, impact on the TRF scale. GGOS Ground Networks and Communications Working Group Meeting, Vienna, ftp://cddis.gsfc.nasa.gov/misc/ggos/0804/ GNCWG_Ries_slrbias_080416.pdf

Rodriguez-Solano CJ, Hugentobler U, Steigenberger P, Lutz S (2012) Impact of earth radiation pressure on GPS position estimates. J Geod 86(5):309-317. doi:10.1007/s00190-011-0517-4

Rubincam DP (1998) Yarkovsky thermal drag on LAGEOS. J Geophys Res 93(B11):13805-13810. doi:10.1029/JB093iB11p13805

Rutkowska M, Jagoda M (2012) Estimation of the elastic earth parameters using slr data for the low satellites STARLETTE and STELLA. Acta Geophysica 60(4):1213-1223. doi:10.2478/ s11600-012-0045-5

Schutz BE, Cheng MK, Shum CK, Eanes RJ, Tapley BD (1989) Analysis of earth rotation solution from Starlette. J Geophys Res 94(B8):10167-10174. doi:10.1029/JB094iB08p10167

Sengoku A (1998) A plate motion study using Ajisai SLR data. Earth Planets Space 50:611-627

Smith DE, Turcotte DL (1993) Contributions of space geodesy to geodynamics: earth dynamics. American Geophysical Union, ISBN 9780875905242, doi:10.1029/GD024

Sośnica K, Thaller D, Jäggi A, Dach R, Beutler G (2012a) Sensitivity of Lageos orbits to global gravity field models. Artif Satell 47(2):35-79. doi:10.2478/v10018-012-0013-y

Sośnica K, Thaller D, Dach R, Jäggi A, Beutler G (2013) Impact of loading displacements on SLR-derived parameters and on the consistency between GNSS and SLR results. J Geod 87(8):751-769. doi:10.1007/s00190-013-0644-1

Sośnica K, Baumann C, Thaller D, Jäggi A, Dach R (2014) Combined LARES-LAGEOS solutions. In: Proceedings of the 18th international workshop on laser ranging, Fujiyoshida, Japan

Sośnica K, Thaller D, Jäggi A, Dach R, Beutler G (2012b) Can we improve LAGEOS solutions by combining with LEO satellites? In: Proceedings of the international technical laser workshop 2012 (ITLW-12), Frascati (Rome), Italy

Thaller D, Sośnica K, Dach R, Jäggi A, Beutler G (2011) LAGEOSETALON solutions using the Bernese Software. Mitteilungen des Bundesamtes fuer Kartographie und Geodaesie. In: Proceedings of the 17th international workshop on laser ranging, extending the range, vol 48, Bad Kötzting, Germany, pp 333-336, Frankfurt, ISBN 978-3-89888-999-5

Thaller D, Sośnica K, Dach R, Jäggi A, Mareyen M, Richter B, Beutler G (2014a) Geocenter coordinates from GNSS and combined GNSSSLR solutions using satellite co-locations. In: Rizos Ch and Willis P (eds) Earth on the edge: science for a sustainable planet, vol 139, International Association of Geodesy Symposia, pp 129-134, doi:10. 1007/978-3-642-37222-3_16

Thaller D, Sośnica K, Mareyen M, Dach R, Jäggi A, Beutler G (2014b) Geodetic parameters estimated from LAGEOS and Etalon data and comparison to GNSS-estimates. J Geod (submitted) 